\title{
LOS COMIENZOS DEL TRANSPORTE MECANIZADO \\ TERRESTRE EN MAGALLANES (1900-1930)
}

MATEO MARTINIC B.

\section{RESUMEN}

Se da cuenta de las formas y circunstancias que determinaron la evolución del sistema de transporte en Magallanes, desde los carruajes de tiro animal propios de la época de la colonización inicial, hasta los vehículos automotores, fenómeno entendido como la culminación del ciclo de modernización mecánica que caracterizó a la crianza ovejera extensiva, que fue la actividad vertebradora matriz de la economía magallánica.

PALABRAS CLAVES: Patagonia, transporte, camiones, tecnología mecánica.

\section{THE BEGINNINGS OF MECHANIZED TERRESTRIAL TRANSPORT IN MAGELLAN (1900-1930)}

\begin{abstract}
The forms and circumstances that determined the evolution of transport systems in Magellan are narrated, from the carriages pushed by animal's characteristic of the initial settling epoch, up to the selfpropelled vehicles, phenomenon understood as the culmination of the cycle of mechanical modernization that characterized the upbringing extensive sheep farming, main vertebrate activity of the magellanic economy.

KEYWORDS: Patagonia, transport, trucks, mechanic technology.

\section{INTRODUCCIÓN}

No cabe duda de que el primer desafío que debieron enfrentar los pioneros al dar inicio y continuidad a la ocupación y poblamiento colonizador

del vasto territorio oriental de Magallanes devenido con propiedad el ecúmene a contar de principio de los años de 1880, fue el de acceder a los diferentes lugares en que fueron surgiendo los establecimientos $y$, una vez en explotación los mismos, el de sacar las
\end{abstract}


correspondientes producciones así como atender a su debido abastecimiento. Si respecto de lo primero, en un comienzo bastaron las cabalgaduras, a poco andar debieron agregarse las carretas de bueyes para transportar desde las caletas o puertos del litoral marítimo más cercano las cargas más pesadas y voluminosas conformadas por materiales y elementos diversos, enseres y muebles, maquinarias, en fin. Tocante a lo segundo, un medio de transporte como la carreta fue una exigencia obligada tanto por razón de su capacidad como por el peso de las cargas ${ }^{1}$.

Es del caso recordar que, dada la configuración fisiográfica interior del territorio regional, esto es, por la interrelación de las zonas terrestres continentales e insulares y las vías marítimas, se facilitó la empresa colonizadora al acercar entre sí, hasta donde fue posible, los lugares de poblamiento con los parajes costeros de entrada y salida por la vía marítima, única disponible desde un principio y que por mucho tiempo sería la más practicable y económica. Pero, así y todo, siempre quedaron sectores situados a gran distancia hacia el interior, de hasta un centenar o más de kilómetros, que debían ser recorridos trabajosamente por los primeros medios de transporte de tiro animal.

Esta forma se mantuvo vigente, con carácter de única o exclusiva, a lo menos durante dos décadas, esto es, hasta el período de cambio entre los siglos XIX y XX, y debió adaptarse en una primera fase a la inexistencia de caminos -los que literalmente debieron hacerse al andar o "abriendo huella" en el hablar común patagónico-y luego, en una segunda etapa, a su precariedad, circunstancia que derivaba de las condiciones de los terrenos por los que transcurrían las sendas, por lo común blandos, y del tamaño y peso creciente de los vehículos. Esto en particular significaba que en vez de un camino en forma como en el presente se las conoce, las vías del tiempo del principio de la colonización eran apenas un conjunto de huellas más o menos paralelas, que en los lugares de más difícil paso se multiplicaban,

1 Aunque genéricamente se hace mención a las carretas (vehículos de dos ruedas tirados por bueyes), es probable que en una época temprana del proceso colonizador circularan también carros (wagons) de cuatro ruedas y tiro cabalgar, inicialmente importados desde los EE.UU. de América o Gran Bretaña y posteriormente fabricados localmente (en Punta Arenas). señalando los esfuerzos por encontrar suelo firme. El transporte terrestre era así extremadamente lento y demoroso, y para ejemplo basta señalar el dato entregado por Rogelio Figueroa, quien en 1903, teniendo en la mano la autorización para ocupar una hijuela industrial en el paraje de Tres Pasos, distrito de Ultima Esperanza, demoró veintitrés días para hacer el trayecto de trescientos kilómetros que media entre Punta Arenas y aquel lugar, con una carreta de bueyes cargada a tope ${ }^{2}$.

El desarrollo de la colonización y la situación de los establecimientos de crianza ovejera con relación a los sitios litorales de embarque/desembarque, cuando aquellos quedaron ubicados en zonas interiores de los diferentes distritos, fue originando la concentración de los flujos del transporte hacia los puntos estratégicamente ubicados para las actividades de carga y descarga. En particular este fue el caso de la importante estancia "Cerro Castillo", en la zona interior del distrito de Ultima Esperanza, a unos sesenta kilómetros del puerto marítimo más cercano. Su posición en una encrucijada de rutas que procedían desde zonas en colonización situadas más al norte, al noroeste, al noreste y al este del establecimiento, tanto en territorio chileno como en el argentino, hizo del mismo un punto de convergencia y de concentración de cargas desde el que salían las tropas de carretas y carros con destino a la costa, Puerto Prat y Puerto Cóndor inicialmente, y Puerto Natales más tarde, o al que arribaban cumpliendo una primera etapa, en el camino de retorno. Dichos lugares, importa señalarlo, distaban entonces dos días de navegación de Punta Arenas, puerto principal de entrada a y salida de la Región Magallánica. Tal circunstancia cobró una nueva relevancia a contar de 1905 cuando la Sociedad Explotadora de Tierra del Fuego, devenida la principal propietaria en el vasto territorio, a uno y otro lado de la frontera internacional, estableció otros dos centros de explotación, la estancia "Cerro Guido", unos cincuenta kilómetros al norte en suelo chileno y "Fuentes del Coyle", aproximadamente veinte kilómetros al oriente, del otro lado de la frontera internacional. Para entender cabalmente lo que se trata, debe tenerse en cuenta que en con-

2 'Apuntes de mi vida', en Documentos Inéditos para la Historia de Magallanes. "Rogelio Figueroa en Ultima Esperanza (1905-1919)". (Memorias de un buscapleitos), Magallania vol. 35(1):139-155. Punta Arenas, 2007. 
junto los tres establecimientos superaban el medio millón de hectáreas en superficie, cabida en la que acabarían pastando no menos de 300.000 ovejas, dotación capaz de entregar unas 1.500 toneladas de carga, lo que en términos de la época era una cifra importante considerada como carga, tanto en peso como en volumen (Fig. 1).

De otra parte, el que la compañía mencionada -la mayor poseedora fundiaria de la Patagonia austral y la Tierra del Fuego, como propietaria (en Patagonia) y arrendataria (en Tierra del Fuego y Patagonia)- dispusiera de suficiente capital para manejar de la manera más eficiente sus intereses y negocios, con posibilidad, además, para prestar servicios a terceros en relación de vecindad, permite situar allí, en el centro neurálgico de Cerro Castillo como apropiado ejemplo, el origen del transporte mecanizado terrestre en Magallanes. Ello fue posible obviamente por la vigencia de una visión moderna en la conducción administrativa de la Sociedad Explotadora de Tierra del Fuego, que valorizaba en lo que correspondía la incorporación de la tecnología mecanizada para hacer más eficiente y económico el transporte de su producción ovina, principalmente de la lana.

Con esta decisión propia de la dinámica evolutiva empresarial de la época se concluiría el ciclo de la mecanización modernizadora en la actividad matriz vertebradora de la economía magallánica, la ganadería ovejera: de la esquila con tijeras a campo abierto, a la operación con cortadoras mecánicas montadas en guías movidas a su vez por un sistema de correas y poleas con fuerza motriz generada por calderas a vapor en grandes galpones industriales; del fundido (por derretimiento) de carcasas ovinas en calderos digestores para la obtención exclusiva de grasa ovina (graserías), a las plantas de faenamiento y frigorización complejas para producir carne y subproductos ovinos para la exportación; y del transporte en carretas, al uso de elementos mecanizados movidos inicialmente por la fuerza del vapor y posteriormente por motores a combustión interna a gasolina. En realidad de verdad, debía esperarse una decisión semejante de parte de la entidad que detentaba el mayor latifundio de la Región Magallánica (3.000.000 hectáreas en 1910), entre terrenos propios y los arrrendados al Fisco, que desde un comienzo se manifestó preocupada por la más eficiente gestión operativa. Su iniciativa trascendente, seguida luego por otras sociedades ganaderas y comerciales acabaría por generar una actividad económica autónoma, el transporte motorizado terrestre, en manos de pequeños empresarios al despuntar la década de 1930 y desde entonces para el futuro.

\section{LA INTRODUCCIÓN DE MEDIOS MECÁNICOS DE ARRASTRE Y TRANSPORTE}

Es sabido que el primer artilugio mecánico automotriz que llegó a Magallanes fue un automóvil marca Star, al parecer con capacidad para el conductor y un pasajero, que en 1903 causó impresión a los habitantes de Punta Arenas muchos de los cuales tal vez ni habían oído mentar tal clase de vehículos. No se sabe qué fin tuvo este automóvil, si es que permaneció en Magallanes, pero sí que pasaron años antes que en las calles de la capital magallánica volvieran a verse los automóviles de manera regular. Esta claro que tal ocurrió en 1914 cuando arribaron tres máquinas destinadas a permanecer: un automóvil marca Napier, importado por A.A. Cameron, otro marca Bedford, traído por el Dr. W. France y un Ford modelo " $\mathrm{T}$ " adquirido por Juan Colorado. Aunque durante los años que siguieron fueron importándose algunos más, todos eran aparatos sencillos y livianos, únicamente de pasajeros.

El primer vehículo del género destinado al transporte de carga debió arribar a Magallanes pasada la mitad de la primera década del siglo en fecha difícil de precisar. El Archivo Fotográfico Histórico del Centro de Estudios del Hombre Austral tiene una tarjeta postal que da cuenta de tal novedad, que muestra a la máquina en la cima del cerro de la Cruz en Punta Arenas, que tiene impresa en su parte superior la leyenda El primer automóvil que llegó a Punta Arenas. En verdad, no fue así, pues como se ha mencionado antes ello ocurrió en 1903, aunque no se discute que el fotografiado fuera efectivamente el primer vehículo automóvil fabricado especialmente para el transporte de carga (propiamente un camión según se lo conoce actualmente) llegado a Magallanes. La sensación pública que causó esa máquina quedó documentada además en otra fotografía de la época, pues se la ve rodeada por vecinos, en tanto que otro grupo posó encaramado sobre la misma (Fig. 6). La marca FIAT (Fabrica Italiana di Automobili Torino) es visible en las protecciones de la camada. En efecto, esa conocida empresa inició su 
producción en 1899 con automóviles de pasajeros, rubro que amplió en 1903 con vehículos para carga, comenzando con un modelo que poseía un motor a gasolina de $24 \mathrm{HP}$ y con capacidad de transporte para cuatro toneladas. En este respecto, nos parece que el vehículo que se muestra en la postal debió corresponder a un modelo posterior ${ }^{3}$.

Como haya sido, si tal máquina pudo despertar la admiración del vecindario puntarenense, también debió concitar la atención y el interés de los empresarios económicos (mercantiles, industriales y ganaderos) que hasta entonces disponían de medios de transporte de carga de tiro animal. Se habrán advertido asimismo las ventajas e inconvenientes de tan novedoso medio mecánico, pues la facilidad del autotransporte y su disponibilidad de carga debieron confrontarse con su costo, quizá elevado, su gran peso bruto, lo que lo hacía poco maniobrable en las vías urbanas no consolidadas, y más en las rurales, y la reducida potencia motriz. Así pues, aunque atractivo e interesante como expresión de modernidad tecnológica, ese vehículo no habría acabado de convencer a quienes lo miraban teniendo en vista el transporte de cargas pesadas en el medio rural.

En ello igualmente debían pensar por ese tiempo los ejecutivos de la Sociedad Explotadora de Tierra del Fuego, a juzgar por una referencia encontrada en la correspondencia de la Administración General con la casa londinense Duncan Fox Ltd., que era su agente comprador en el Reino Unido. En efecto, en un postscriptum de una carta fechada el 6 de junio de 1906, tras el epígrafe Steam Wool Wagon, se expresa textualmente, Cuando visitamos la Exhibición Real [Royal Show] en Derby la semana pasada tomamos un tiempo en averigüar respecto de los Steam Wool Wagons. Los Sres. Poden Ltd. son los mejores fabricantes de esta clase de carros y ellos prepararon para nosotros la propuesta de un vehículo que probablemente se ajusta a nuestros requerimientos ${ }^{4}$.

3 Una de las vistas fue tomada desde el recinto donde por entonces se construían los estanques de almacenamiento del servicio de agua potable de Punta Arenas, cuyos trabajos se realizaron entre 1906 y 1908, lo que permite situarla cronológicamente hacia este último año.

4 Correspondencia con la casa Duncan Fox año 1906. Carpeta V, Fondo S.E. T.F. Archivo de Documentos Inéditos, Centro de Estudios del Hombre Austral, Instituto de la Patagonia, Universidad de Magallanes, Punta Arenas.
La propuesta se refería a un modelo incluido en un catálogo ilustrado de la firma fabricante que se acompañaba a la carta, que se denominaba spring mounted trailer para una carga de tres toneladas, provisto con una plataforma plana de 12 pies de largo por 6 de ancho, ruedas de 3 y de 3 pies 6 pulgadas de diámetro por 9 de ancho en la superficie de rodamiento, a un costo FOB de cincuenta libras esterlinas la unidad. Así, debe entenderse que la cotización se refería únicamente a una plataforma rodante para carga, pues no hay mención a la máquina de tracción. Ello permite suponer que hubo alternativas de adquisición que favorecieron a otro proveedor. De esa manera, en una fecha indeterminada que debiera situarse por ese tiempo la mencionada compañía importó desde Gran Bretaña las primeras unidades mecanizadas de arrastre, o sea tractores a vapor, adquiridos a la prestigiada firma fabricante J. \& H. Mc Laren Ingeneers, de Leeds, para el servicio de sus estancias en Patagonia y Tierra del Fuego, siendo posible que su número no bajara de cuatro. De estas interesantes máquinas, hoy con un valor histórico patrimonial, se conserva una (la número 1296) en el Museo del Recuerdo del Instituto de la Patagonia, Universidad de Magallanes, en la ciudad de Punta Arenas (Fig. 2).

Esta adquisición fue complementada con otra correspondiente a plataformas rodantes (Platform trucks) con capacidad para cargar hasta siete toneladas (no menos de doce fardos de lana), según una orden de compra de cuatro unidades cursada en Punta Arenas el 13 de junio de 1908, que hemos tenido a la vista ${ }^{5}$. Los carros, de acuerdo con las especificaciones requeridas, debían tener frenos pero no resortes y ser fabricados para moverse sobre caminos de arcilla, por lo que no debían soportar vibraciones como sucedía en los caminos pavimentados con macadam en el Reino Unido.

Se deseaba conformar así un tren de transporte mecanizado con un tractor y entre tres y cuatro carros, es decir, con una carga neta de arrastre de hasta treinta toneladas de lana. La velocidad de desplazamiento era de cuatro kilómetros por hora, a base del dato obtenido de la correspondencia, para el caso una carta del administrador de Cerro Castillo a la Administración General. En la misma se da cuenta de que el menor tiempo empleado entre el casco de esa estancia y

5 Sociedad Explotadora de Tierra del Fuego, Copiador de Cuentas Sección Ultima Esperanza (Marzo 1906-Marzo 1910), archivo citado. 
Tres Pasos, paraje situado a unos 30 kilómetros de distancia, había sido de siete y media horas, y que el resto del viaje había tomado otras seis y media horas ${ }^{6}$. En buenas cuentas, la operación tomaba más de un día y a juicio del administrador un conductor diligente con una carga de entre once y doce toneladas debía demorar un día. Este tren de transporte se operaba con un maquinista y un ayudante (Fig. 3).

Así entonces se inició y mantuvo por unos tres lustros un servicio de transporte mecanizado de carga, de lana principalmente, desde el establecimiento mencionado hasta el puerto de embarque, que en un principio fue Puerto Cóndor, situado en el acceso al fiordo Eberhard, pero que hacia 1910 fue reemplazado por Puerto Bories, factoría industrial de la misma compañía mencionada, que tenía un buen muelle para el atraque de naves mayores tanto del cabotaje regional como de las procedentes de ultramar.

Un viajero de la época, el capitán de ejército Arturo Fuentes Rabé, que recorrió en 1918 la isla grande de Tierra del Fuego, pudo observar el mismo sistema de transporte mecanizado en ese distrito, consignando [...] un pito igual al de una locomotora, tocado a cierta distancia de Puerto Nuevo, llama hacia tierra nuestra atención. Por el espléndido camino [sic] que se dirige a las casas de la estancia [Caleta Josefina], avanzan enormes tractores que arrastran varios carros cargados con numerosos fardos de lana. Vistos desde lejos, semejan trenes completos corriendo por sobre terraplenes sin rieles ${ }^{7}$.

En la misma época de que se trata, aunque en fecha indeterminada, la Sociedad Explotadora de Tierra del Fuego importó para sus necesidades de transporte otro tipo de máquinas automóviles, para el caso calderas rodantes, semejantes en aspecto a un camión moderno, esto es, con plataforma incorporada y trasmisión a cadena. La plataforma, en el caso de distancias largas parece haberse utilizado en parte para la carga del combustible que alimentaba a la caldera (leña o carbón). La máquina actuaba como elemento de tracción de un tren compuesto por carros de arrastre, del mismo modo que los tractores Mc Laren. Hacía el recorrido entre la estancia "Oazy Harbour", en la zona centro-oriental de Magallanes, y el puerto del mismo nombre, en

6 Carta de 15 de diciembre de 1908, en correspondencia y archivo citados.

7 Tierra del Fuego (Valdivia, 1923), tomo II, pág. 19. la costa del Estrecho. De esta clase de máquinas hubo a lo menos dos en servicio, una de las cuales fue rescatada del basural de la estancia en 1971 y trasladada al Museo del Recuerdo del Instituto de la Patagonia, donde se exhibe con el carácter de pieza única en su tipo (Fig. 4).

Sin perjuicio de la operación del sistema de transporte descrito, los ejecutivos de la Explotadora atentos al adelanto que de tanto en tanto se registraba en la materia y teniendo en consideración la incorporación de elementos de tracción más veloces y eficientes, consideraron la conveniencia de importar máquinas con motor a gasolina conocidas como trucks (camiones).

\section{LLEGADA Y OPERACIÓN DE LOS PRIMEROS CAMIONES}

Aunque no tenemos certidumbre acerca de la fecha precisa -año- para la introducción de los primeros vehículos de carga autopropulsados, esto es, camiones como comúnmente se los conoce, debe aceptarse que ello sucedió hacia 1910, más bien entrada la segunda década del siglo. Como cabía esperarlo, la Sociedad Explotadora de Tierra del Fuego -a la que atribuimos la iniciativa-, siempre atenta a la modernidad mecánica hubo de considerar tempranamente la posibilidad de incorporar ese tipo de máquinas, sin perjuicio de mantener operativo su sistema de transporte con tractores Mc Laren.

La búsqueda y selección del modelo más recomendable debió concluir con la adquisición a la Albion Motor Car Company Limited, de Scotstoun, Glasgow, que gozaba de renombre por la excelencia de su producto, exclusivamente camiones, que venía fabricando desde 1899. Así, aunque no se sabe la cantidad de vehículos que pudo comprar, sí consta que ya eran utilizados a principios de 1917 en Última Esperanza. La información, para el caso, la brinda un certificado de 29 de marzo de 1917 emitido por el Dr. Ildefonso Díaz y Pereyro, médico de la compañía contratado para el distrito, que da cuenta de la imposibilidad de embarque de un trabajador enfermo en el vapor Alejandro debido al retraso del tractor auto Albion que lo traía, por causa del mal estado del camino ${ }^{8}$.

8 Carpeta Problemas Laborales y Accidentes del Trabajo, Años 1916-1917, fondos citados. 
A la vista de las únicas fotografías conocidas de estos vehículos, hacemos una descripción somera del modelo adquirido: una máquina de chassis corto, ruedas macizas (las traseras dobles); ñato, es decir con la cabina abierta sobrepuesta, dejando espacio para el asiento del conductor y de un ayudante, y motor con un gran radiador. Su capacidad de carga era de unas tres toneladas y media (diez fardos de lana de 300 kilos aproximadamente cada uno); con fuerza de arrastre para un carro auxiliar que podía llevar ocho fardos, lo que supone una capacidad de carga de seis toneladas. $\mathrm{Su}$ menor capacidad de carga, comparada con la de los tractores antes mencionados se compensaba con la mayor velocidad que brindaba su motor a gasolina, lo que significaba que en igual período de tiempo al que empleaban éstos, los camiones podían hacer mayor cantidad de viajes y posiblemente con mayor economía en la relación tiro-carga-combustible (Fig. 7). Su período de uso fue extenso, quizá superior a tres décadas, posiblemente hasta 1950 , es decir hasta que por una parte la obsolescencia de las máquinas, unida a la escasez o falta de repuestos, y a la externalización del servicio de transporte que consta hizo la Sociedad Explotadora hacia la mitad del siglo, determinaron su abandono?.

Para entonces la noción acerca de la utilidad práctica de los auto-camiones se había generalizado como para que un pequeño empresario hotelero y también ganadero como era Rogelio Figueroa, ya mencionado, pensara en una eventual adquisición. Así hemos podido constatarlo por una nota de fecha 5 de febrero de 1913 que obra en su correspondencia y dirigida a la firma comercial importadora Auby Company, manifestándole su interés por comprar un camión automóvil con capacidad de 3 ó 4 toneladas. Que sea aparente en caminos con gradiente y la mayor parte del año con barro en los caminos ${ }^{10}$.

$9 \quad$ El ingeniero señor René Venegas, quien en 1957 se hizo cargo de la dirección de la maestranza de la estancia "Cerro Castillo", nos ha informado que entonces se mantenían en uso dos camiones "Albion", aunque para el transporte de leña en trechos cortos. Estas máquinas fueron dadas de baja hacia 1960-61 y en manos de terceros prolongaron su vida útil por un tiempo más dedicados al transporte de madera en la zona de Puerto Natales.

10 Copiador de Cartas de Rogelio Figueroa, folio 64, archivo citado.
Que tal innovación podía resultar un fracaso lo prueba el hecho de la importación hecha por Juan Rudd, propietario de la estancia "Cabo Buen Tiempo", en el vecino Territorio de Santa Cruz (Argentina), quien compró en 1912 un camión "Commer", con ruedas traseras de hierro que utilizaba keroseno como combustible, pero que no dio resultado ${ }^{11}$.

La información más confiable que permite situar cronológicamente el comienzo del transporte de carga en camiones la encontramos en la Revista del Camino 1941, publicación hecha por el antiguo Departamento de Caminos (actual Dirección de Vialidad del Ministerio de Obras Públicas), al señalar el año 1913 como época de la circulación del primer camión a vapor por los caminos magallánicos.

Corriendo el tiempo diversas agencias mercantiles del ramo establecidas en Punta Arenas pudieron ofrecer modelos de diferentes marcas para la importación, tales el "Overland" (hacia 1915-18), el "Bedford" (hacia 1918-20), también "Four Wheel Drive" y "Nash Quad", todos de fabricación inglesa, chassis sin cabina y ruedas macizas de hierro, aunque no se sabe si eran movidos por motores de vapor o gasolina; $y$, por supuesto, el camión fabricado por la Ford Motor Company de los Estados Unidos de América, producto interesante pero liviano y de capacidad de carga reducida. En el álbum Punta Arenas y Magallanes, editado por el fotógrafo y pintor Carlos Foresti en 1920, se pueden observar dos tipos de camiones en las calles de Punta Arenas -todavía una rareza-, uno al parecer de tipo liviano con motor a gasolina y ruedas con neumáticos (¿Ford?), estacionado frente a la casa matriz de Braun \& Blanchard, y otro con motor a vapor, ruedas de hierro (de mayor diámetro las traseras) y trasmisión a cadena, cargado a tope con cajas de licores fabricados por Depetris y Cía. (Fig. 5).

Como ocurrió con otros inventos mecánicos de data relativamente reciente, la Gran Guerra Europea (Primera Guerra Mundial) en su transcurso entre 1914 y 1918 influyó directamente en su progresivo perfeccionamiento a través de innovaciones tecnológicas y combinación de invenciones mecánicas y de otro género. Con ello, en lo que atañe al transporte

11 D. Osvaldo Topcic, Historia de la Provincia de Santa Cruz. Crónicas y Testimonios (Centro de Estudios Históricos "Prof. S.A. Segreti”, Córdoba), pág. 146, Córdoba, 2006. 
terrestre automóvil, se tuvo al cabo del conflicto un adelanto sustancial en aspectos tales como el motor de combustión a gasolina o petróleo para la generación de la fuerza impulsora, el mayor tamaño y la envergadura de los vehículos y, particularmente, el agregado de una capacidad de carga importante tanto en volumen como en peso. Así entonces, con la postguerra los camiones, trucks, se incorporaron de lleno a la vida civil cotidiana, directamente al servicio de la economía productiva como un medio de transporte urbano y rural que se haría cada vez más indispensable.

En efecto, la utilidad y versatilidad de los autocamiones quedaron probadas tanto en los campos de batalla europeos, como en las circunstancias de la crisis norteamericano-mexicana de 1916, oportunidad en que estos vehículos fueron empleados en zonas naturales muy difíciles desprovistas de vías camineras, desafío al que respondieron exitosamente, lo que contribuyó a cimentar su fama e hizo del ejército de los EE.UU. de América un importante comprador ${ }^{12}$.

Las ofertas para el mercado consistían en modelos de autocamiones con capacidad de entre una y media y seis toneladas de carga. Se trataba básicamente sólo del chassis de cada máquina, motor incluido, quedando la elección del tipo de la plataforma y la caja al gusto o la necesidad de los compradores potenciales según el uso que deseara dárseles (plataformas fijas o de volteo; rejas protectoras de carga; cabinas y toldos, y otros tipos). De cualquier modo, esa oferta debió ser restringida a juzgar por la ausencia de referencia al rubro en el avisaje comercial de los diarios puntarenenses de la época (segunda década del siglo).

$\mathrm{Su}$ incorporación creciente en el servicio de transporte en Magallanes se dio, está claro, a fines de la segunda década y, otra vez, advertida que fue su maniobrabilidad y versatilidad de empleo, como su capacidad de respuesta a las exigencias de los precarios caminos de entonces. De esa manera, poco a poco los pesados vehículos movidos por la fuerza del vapor fueron cediendo lugar a los nuevos, los autocamiones, más livianos y maniobrables.

12 The Nelly-Springfield Motor Truck C., Autocamiones NellySpringfield (Catálogo), Springfield, 1917. Archivo Museo Regional de Magallanes, Punta Arenas.

\section{LA GENERALIZACIÓN EN EL USO DE CAMIONES}

La tercera década del siglo XX fue el período histórico de consagración de la modalidad de transporte mecanizado automotriz en Magallanes. En efecto, la postguerra trajo la anhelada paz, que fervientemente se esperaba que fuera duradera por toda la humanidad, pero también puso a disposición de todo el mundo para fines de progreso y bienestar los diferentes productos que la tecnología mecánica perfeccionada por la inventiva de los antiguos beligerantes había desarrollado durante los años del conflicto mundial. Entre tantos productos industriales, y en lugar calificado por cierto, estaban los automotores destinados al transporte de carga. De esa manera, la oferta se hizo variada en lo tocante a modelos, calidades y precios, en una evolución tecnológica incesante hacia la modernidad.

Así, pasado 1920 fueron llegando a Magallanes camiones livianos de la fábrica Ford y también algunos chassis de otras marcas con plataformas que se adaptaron para el transporte de pasajeros. Que esta modalidad deviniera por un buen tiempo la más conveniente, lo prueban dos avisos aparecidos en el diario El Magallanes del mes de setiembre de 1929, en los que Braun \& Blanchard (agentes de Ford para Magallanes) y Arecheta Hnos. ofrecían, respectivamente, chassis de camión con trasmisión auxiliar y chassis de $3 / 4$ toneladas marca "Dover". La versatilidad de la modalidad -sólo chassis con plataforma- permitía su transformación en carros bomberiles, buses de pasajeros y camiones de carga propiamente tales, aunque todavía restringidos al uso del ámbito urbano, para el caso principalmente en Punta Arenas y en Puerto Natales. Es que, por algunos a lo menos, se miraba con recelo a estas máquinas automóviles porque consideraban que destruían los caminos. Tal fue, precisamente, el planteamiento de Juan Agustín Yañez, uno de los miembros titulares de la Junta de Alcaldes del Territorio (organismo municipal único) al oponerse durante el segundo semestre de 1928 a una petición de exención de pago de patente por lo que restaba del año elevada por Antonio Sancho para sus camiones hacía poco importados.

Pero los verdaderos camiones no tardaron en aparecer y en manifestarse como los vehículos apropiados para el transporte de carga intra y ultra 
urbano lo que sucedió al promediar la década. Los primeros adquirentes pasaron a ser otros tantos empresarios del ramo, siendo posible que Sancho Hnos. fuera la entidad inicial de la actividad, particularmente en el ámbito rural. Consta que ya operaba en Punta Arenas en 1927 y que en el curso del año siguiente importó seis camiones Dodge con sus respectivos acoplados, lo que debió darles una capacidad de carga que estimamos fuera de entre seis y ocho toneladas en conjunto para cada uno. Fue esta firma, al parecer, la primera en establecer el servicio de transporte de los productos de la ganadería desde las zonas rurales hasta el puerto de Punta Arenas (Fig. 8). Por entonces, también el empresario Carlos Fischer estableció su empresa de transporte de carga, con tres camiones que al parecer eran de la marca "Mercedes Benz", con capacidad de hasta cuatro toneladas de carga, que igualmente se destinaron al movimiento rural, principalmente en el transporte de lanas y madera elaborada. Más tarde todavía, y con la probable omisión de otros empresarios, se dedicaron a ese tipo de actividad los hermanos Marcos y Alberto Harambour, con un camión marca "Internacional", y los hermanos Alfredo y Rodolfo Novoa que sin perjuicio de mantener el transporte de pasajeros entre Punta Arenas y Puerto Natales, iniciado en 1923, en el cambio de décadas ampliaron su actividad al rubro de carga empleando para ello tres camiones Ford con capacidad de 3/4 toneladas cada uno, principalmente como prestadores de servicio para la Sociedad Explotadora de Tierra del Fuego.

El uso de camiones livianos para servir como buses para el transporte de pasajeros por caminos rurales se registró durante la primera mitad de la década de 1920, inicialmente en recorridos cortos, como fue el caso de la empresa de Armando Santucci que estableció un servicio regular diario entre Punta Arenas, Río Seco y Chabunco.

Estos antecedentes llevan a suponer que la importación de camiones pudo alcanzar alguna importancia en el comercio especializado de Punta Arenas, pero en verdad ello sucedió recién pasado 1930. Con todo, la revisión de la prensa de la capital territorial de la época permite ver una suerte de competencia entre las diferentes agencias importadoras para la captación de clientes, como lo sugiere una información, a modo de aviso pagado, aparecida en el diario El Magallanes de 21 de julio de 1928, referida a la adquisición reciente de camiones "Mercedes Benz" por la Junta de Alcaldes de Magallanes para las obras de pavimentación en Punta Arenas, por ser el vehículo que más rinde, tanto en capacidad como en considerable economía de consumo. Agente de la marca para el Territorio era la firma Baer y Landolt.

En Puerto Natales el primer camión de carga del que hay memoria fue un modelo Ford adquirido para el servicio de la sucursal local de la casa Braun \& Blanchard y que comenzó a circular hacia 1924 ó 1925 para el traslado de mercaderías entre sus bodegas, los muelles del puerto y el Frigorífico Bories. En 1926 este vehículo fue vendido a la empresa de carretas Díaz y Pardo, formada por Fidel Díaz y Jorge Pardo Núñez, que de esa manera modernizaron el servicio que prestaban en el transporte de lana, madera, abono y otros. Antiguos vecinos recordarían después el revuelo que su funcionamiento causó en un principio en la población, que se manifestaba en el interés con que se seguían sus movimientos, en las frecuentes visitas al galpón donde se guardaba para observarlo con mayor tranquilidad y la petición para hacer un paseo arriba del mismo por las irregulares calles de la pequeña ciudad ${ }^{13}$.

Además de camiones de las marcas mencionadas, hacia el fin de la década se movían vehículos "Mack", "Nash", "Diamond", "Fargo D 900", "Thornycroft" y otros por el extenso ámbito sudpatagónico oriental, que barrieron con las carretas y sus boyadas, en acertada frase del historiador Topcic ${ }^{14}$. Esta referencia es especialmente válida porque por sobre la separación fronteriza las realidades geográficas, las explotaciones económicas y los usos tecnológicos consiguientes conformaban un patrimonio común para Magallanes y Santa Cruz.

Efectivamente, con el término de la tercera década del siglo XX, o mejor, con el advenimiento de la siguiente, el transporte mecanizado con vehículos automóviles provistos de motores a gasolina acabó por generalizarse e imponerse en Magallanes, estimulado tanto por su eficiente rendimiento para el movimiento de cargas, como por el progresivo mejoramiento de los caminos cada vez más valorizados por la comunidad para la mejor comunicación intrarregional y, por ende, para el beneficio general.

13 Antecedentes proporcionados por el señor Nelson Alvarez, que agradecemos.

14 Op. cit., pág. 62. 
Con este fenómeno de emergencia del transporte mecanizado se fue registrando de manera coetánea la declinación del antiguo sistema combinado basado en el uso de las carretas y de los barcos, que perdería toda vigencia al promediar los años de 1930.

Concluía así un ciclo histórico de la economía territorial referido al transporte y se iniciaba el posicionamiento definitivo de una nueva modalidad expresiva de la modernidad tecnológica del tiempo. Con el cambio, asimismo, mutaron los protagonismos correspondientes, pues si en el antiguo sistema el transporte había sido una operación complementaria propia del gran empresariado productor o prestador de servicios (y como tal partícipe o integrante de los grandes holdings económicos), en el nuevo, a partir de 1930, la actividad sería asumida generalmente por empresarios independientes medianos y pequeños, circunstancia que caracterizaría social y económicamente el cambio de que se trata. Todo un anticipo, además, de la mutación que se advertiría en la economía magallánica pasada la mitad del siglo XX en la gran mayoría de los diferentes ámbitos productivos y de servicios.

\section{FUENTES DE CONSULTA}

\section{a) Inéditas}

Apuntes de Alfredo Novoa C.

Carpeta Problemas Laborales y Accidentes del Trabajo. Años 1916-1919.

Fondo Sociedad Explotadora de Tierra del Fuego, Archivo de Documentos Inéditos Centro de Estudios del Hombre Austral, Instituto de la Patagonia, Universidad de Magallanes, Punta Arenas.

Correspondencia con la casa Duncan Fox año 1906. Fondo Sociedad Explotadora de Tierra del Fuego. Id. id.

Correspondencia Sección "Cerro Castillo" con Administración General en Punta Arenas. Años 1906-08-09-11. Fondo Sociedad Explotadora de Tierra del Fuego. Id. Id.

Copiador de Cuentas Sección Ultima Esperanza (Marzo 1906 - Marzo 1910). Fondo Sociedad Explotadora de Tierra del Fuego. Id. id.

Copiador de Cartas Rogelio Figueroa. Id. id. b) Impresas

DEPARTAMENTO DE CAMINOS. 1941. Revista Día del Camino 1941. Punta Arenas.

DIARIO EL MAGALLANES. Números sueltos década de 1920. Punta Arenas.

FUENTES RABE, ARTURO. 1923. Tierra del Fuego. Valdivia.

MARTINIC B., MATEO. 19. El encanto de las antiguas máquinas a vapor. Infórmese №64. Empresa Nacional del Petróleo. Punta Arenas.

MARTINIC B., MATEO. 2006 (1992). Historia de la Región Magallánica. Ediciones de la Universidad de Magallanes. Punta Arenas.

MARTINIC B. MATEO. 2007. Documentos Inéditos para la Historia de Magallanes. "Rogelio Figueroa en Ultima Esperanza” (1905-1919). (Memorias de un buscapleitos). Magallania, vol. 35(1): 139-155. Punta Arenas.

REVISTA “MENENDEZ BEHETY”, Punta Arenas 1930.

THE KELLY SPRINGFIELD MOTOR TRUCK Co. 1917. Catálogo. Springfield.

c) Iconografía

FORESTI, CARLOS. 1920. Album Punta Arenas y Magallanes. Punta Arenas.

Archivo Fotográfico Centro de Estudios del Hombre Austral, Instituto de la Patagonia, Universidad de Magallanes, Punta Arenas.

Museo del Recuerdo, Centro de Estudios del Hombre Austral. Id. id. 


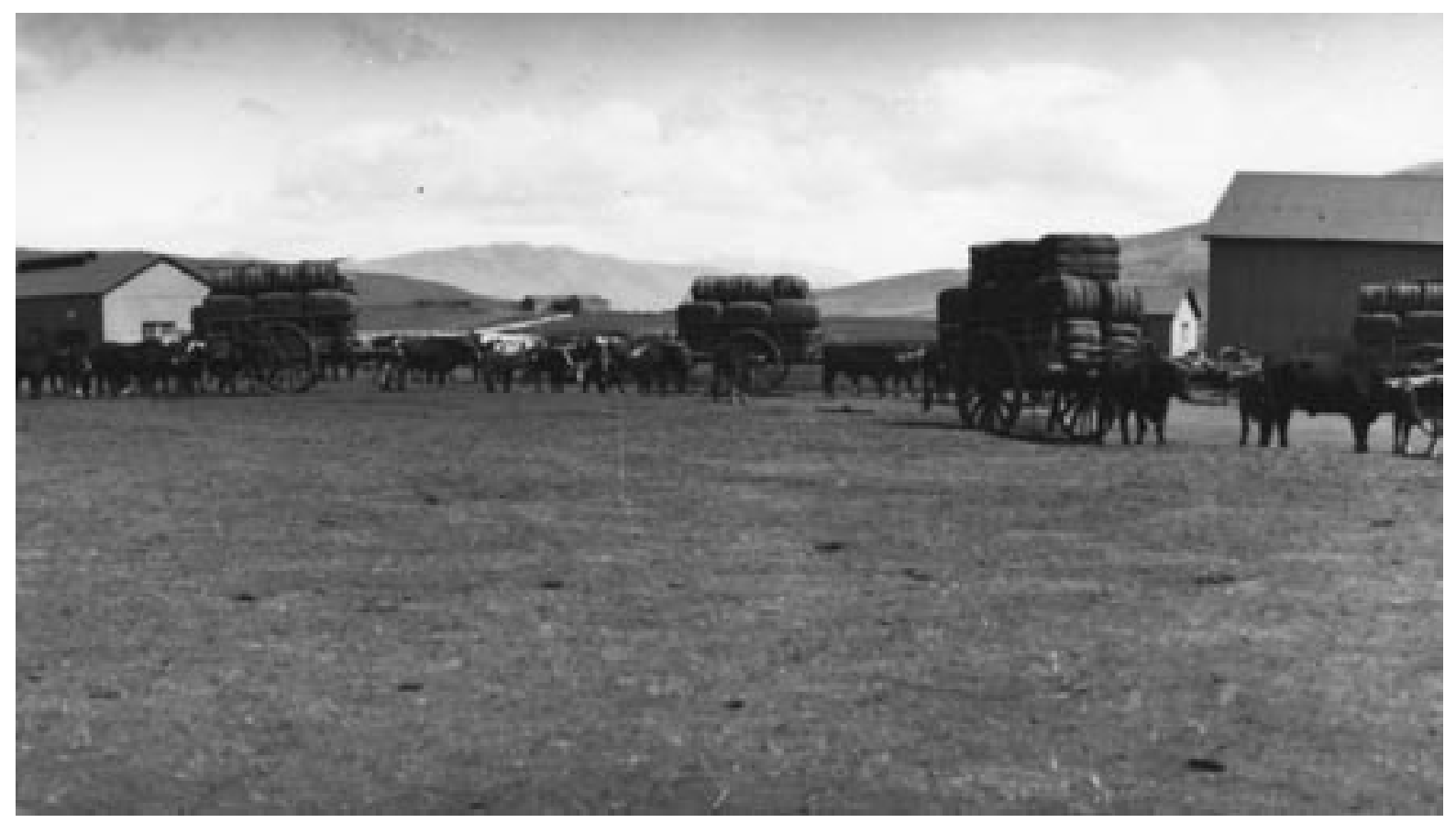

Fig. 1. Carretas de lana en la estancia "Cerro Castillo".

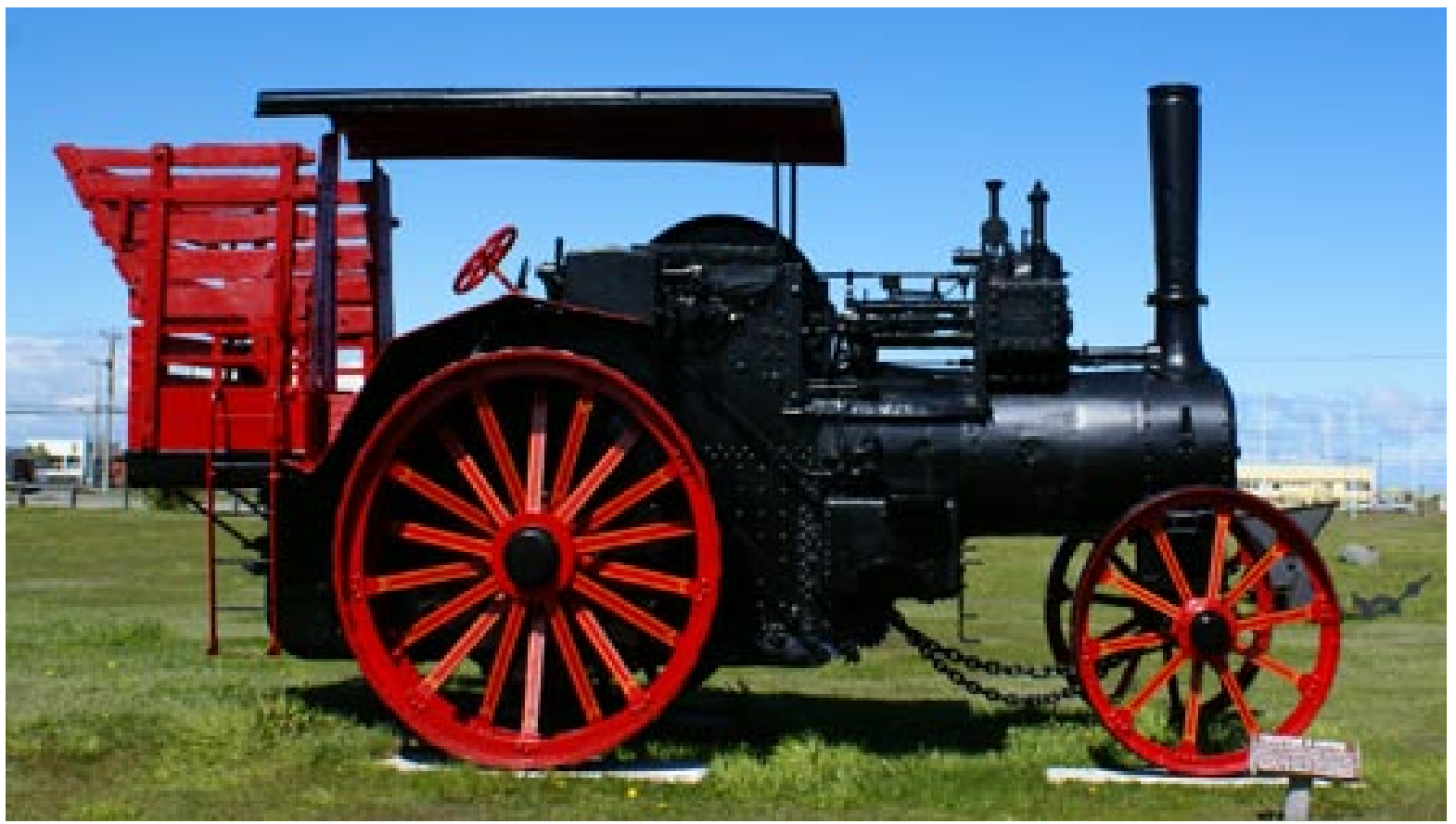

Fig. 2. Tractor Mc Laren, Museo del Recuerdo (Instituto de la Patagonia, Universidad de Magallanes). 


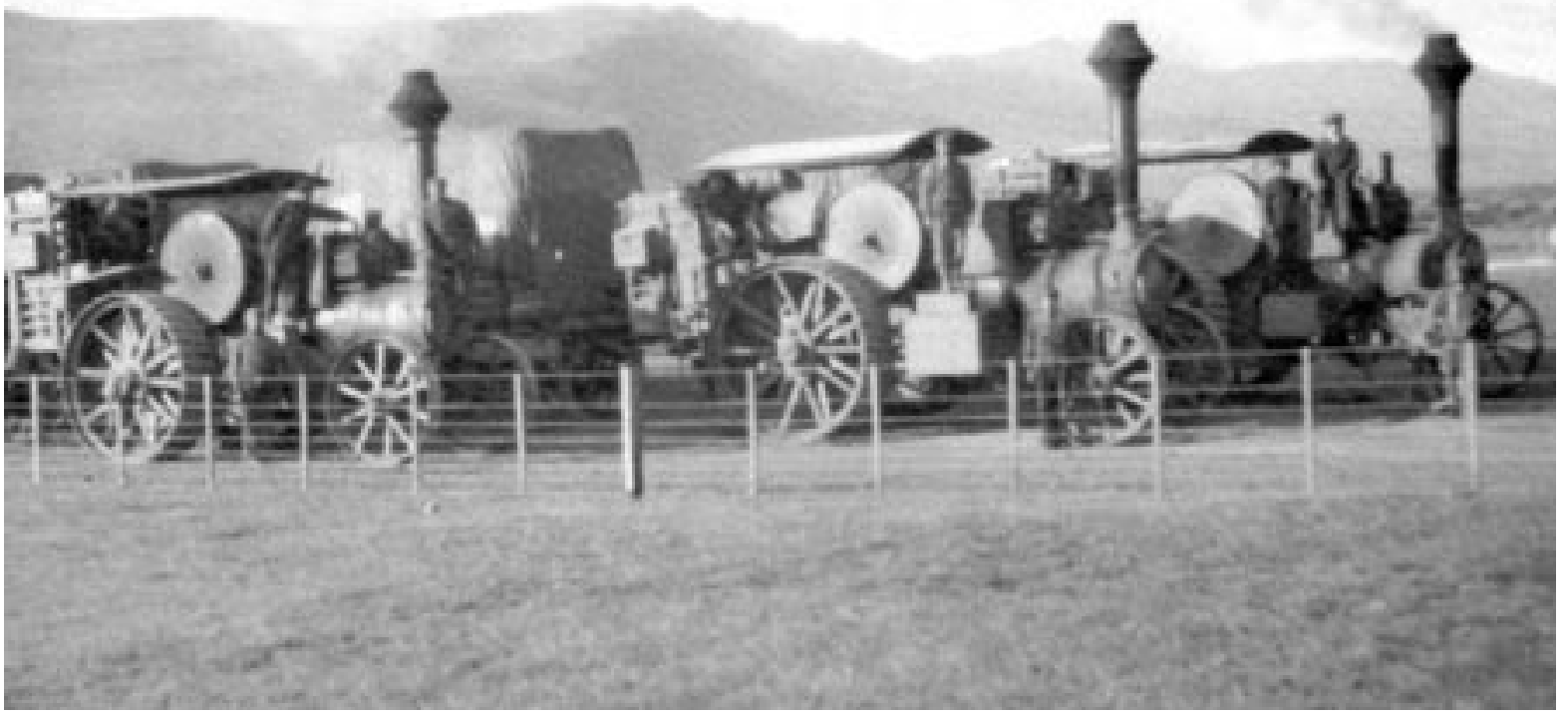

Fig. 3. Tractores y acoplados con carga, estancia "Cerro Castillo".

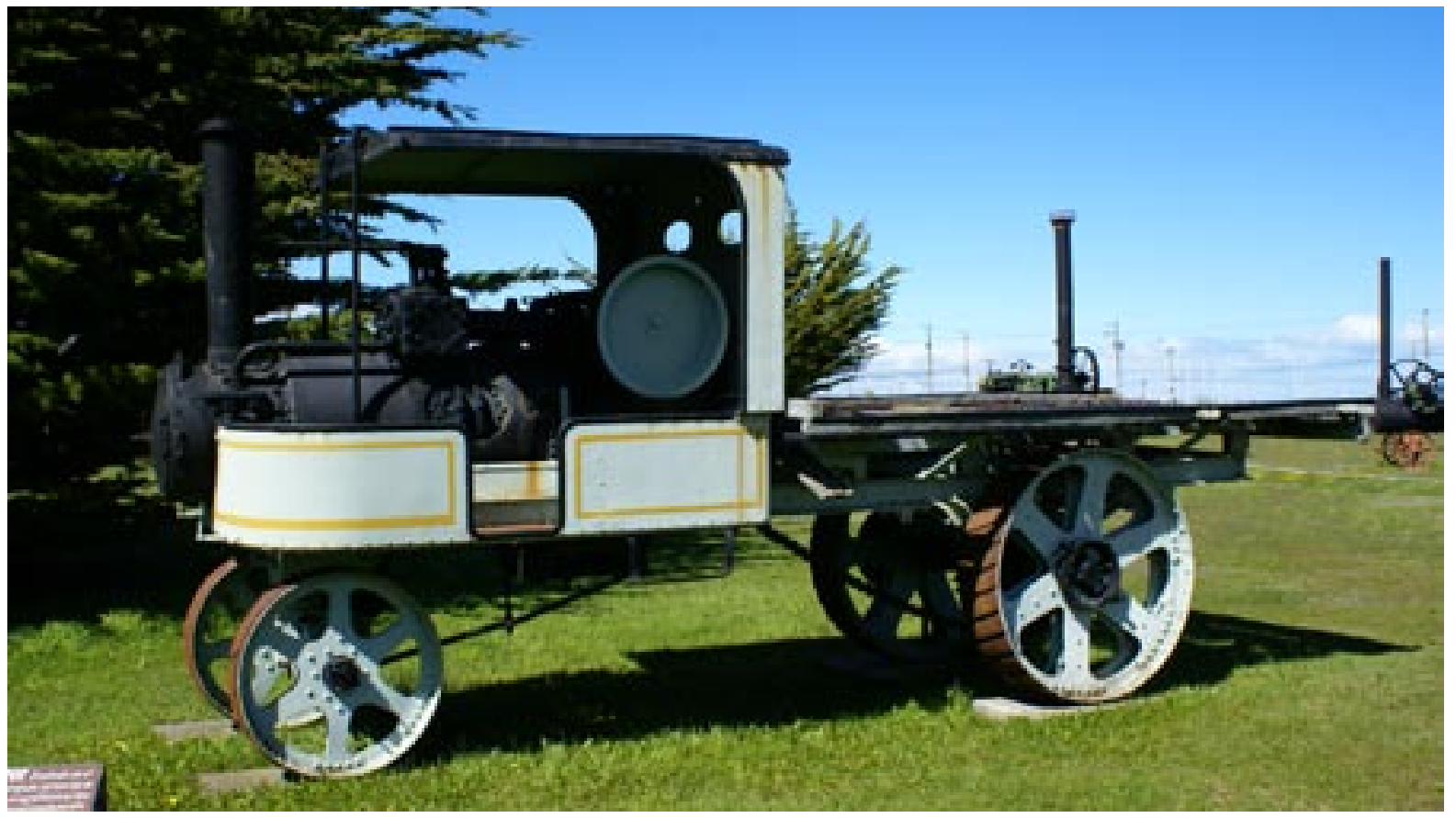

Fig. 4. Camión a vapor, Museo del Recuerdo (Instituto de la Patagonia, Universidad de Magallanes). 


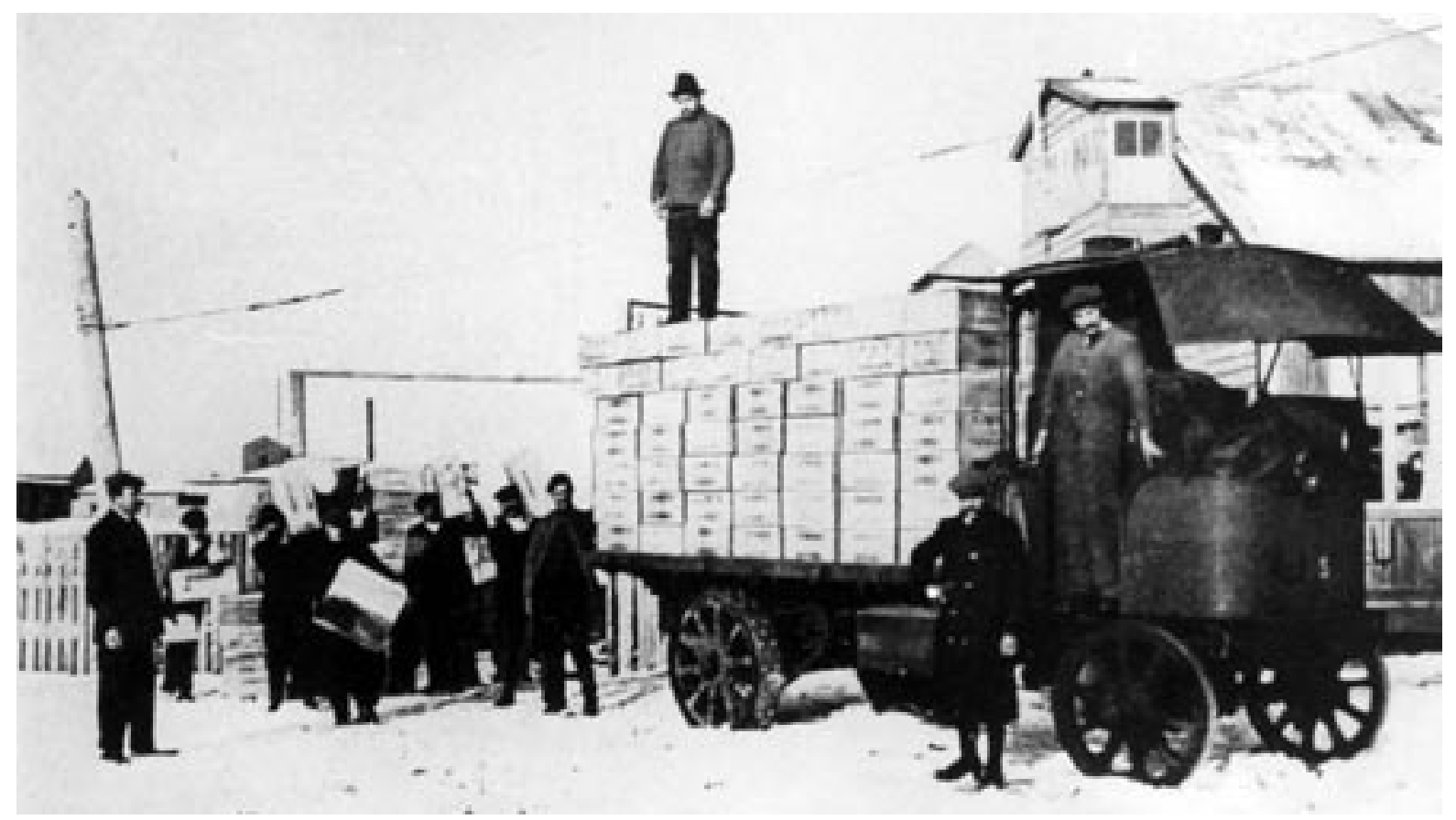

Fig. 5. Camión a vapor en Punta Arenas hacia fines de los años 1910.

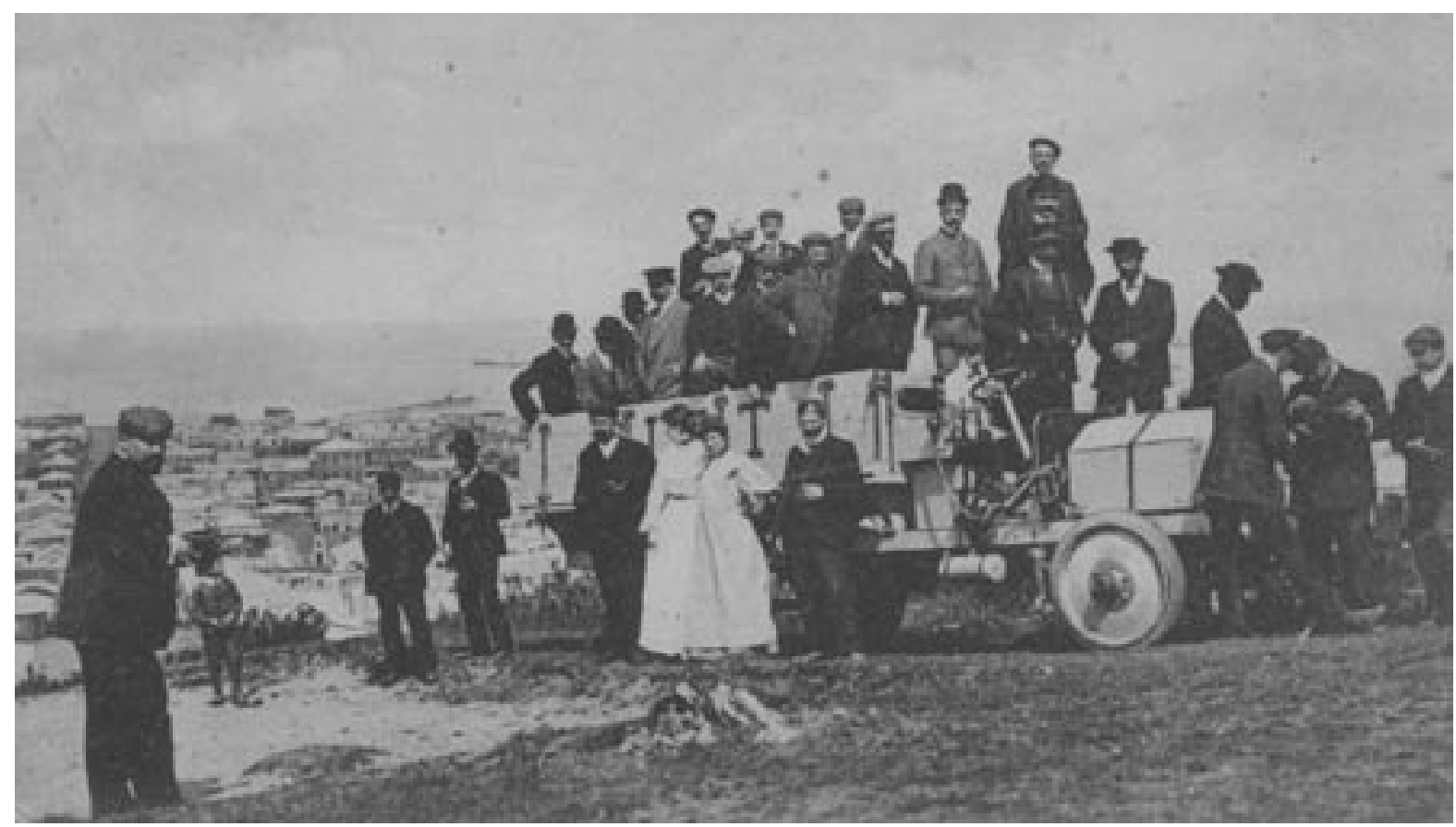

Fig. 6. Auto-camión "Fiat", el primer vehículo de su género que habría llegado a Punta Arenas, posiblemente antes de 1910. 


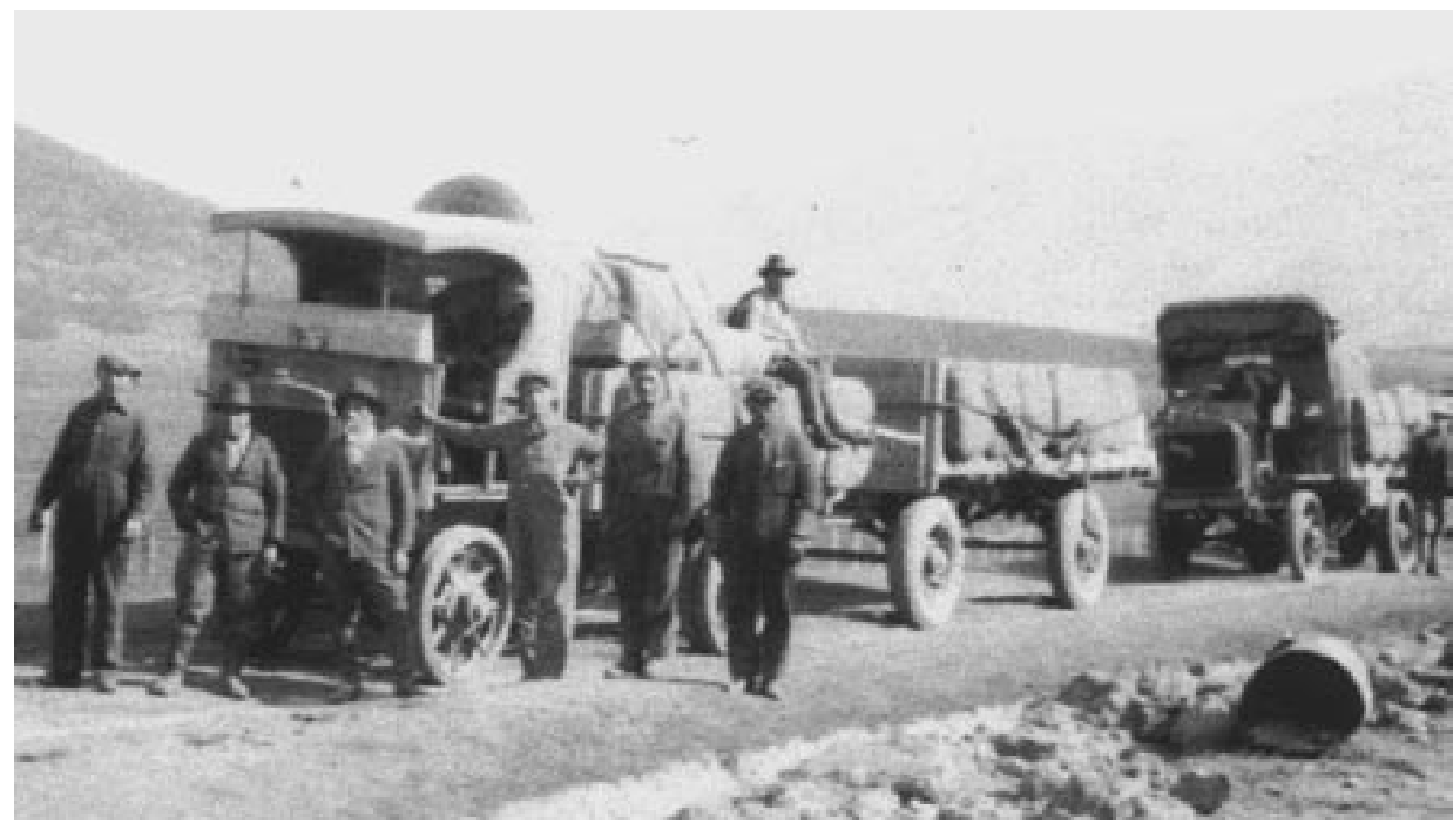

Fig. 7. Camiones con motor a gasolina y sus acoplados en ruta desde la estancia "Cerro Castillo" a Puerto Natales. Fotografía de época indeterminada de los años de 1920.

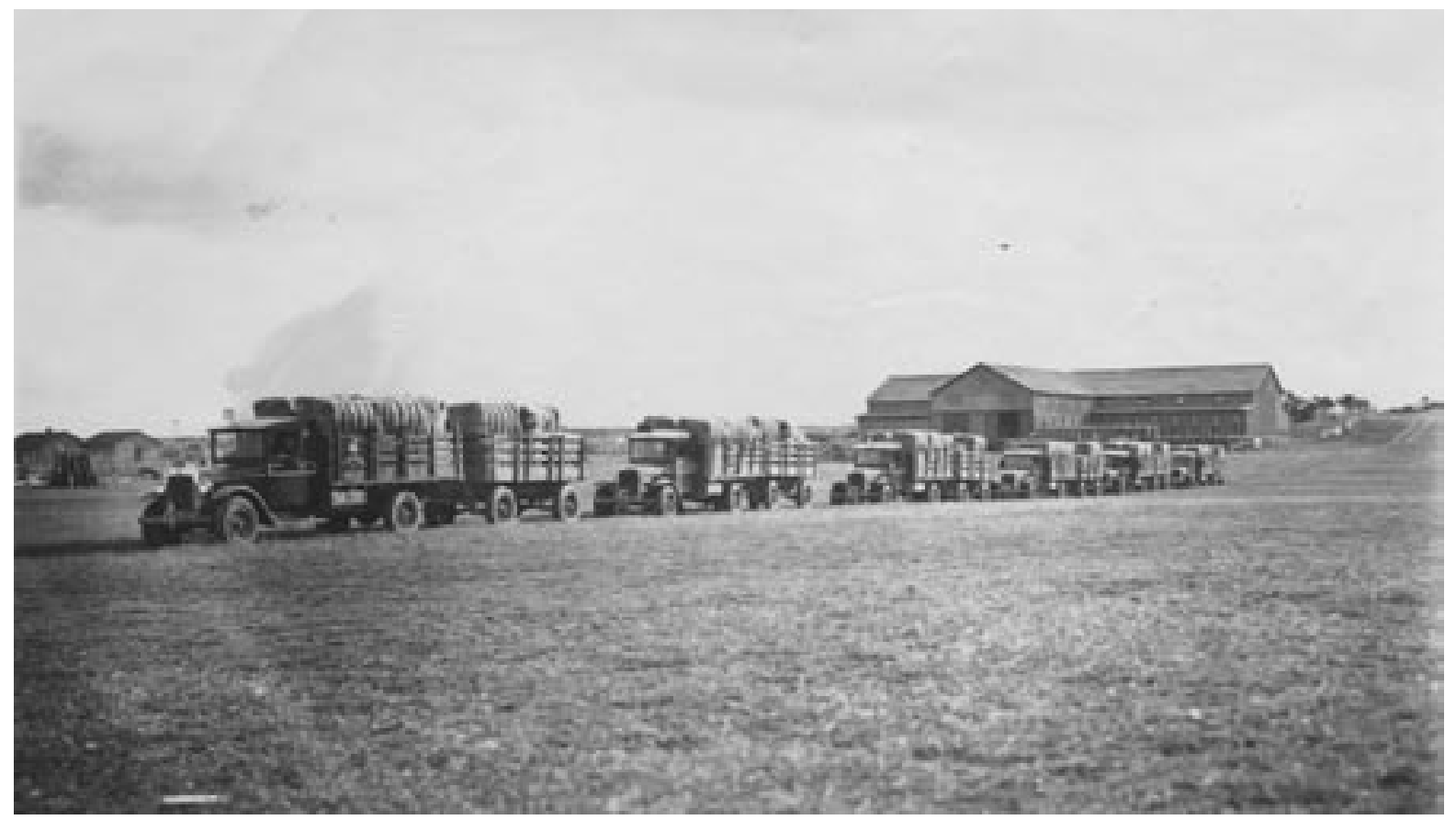

Fig. 8. Camiones "Dodge" de la empresa Sancho Hnos. (1930). 
MATEO MARTINIC 